\title{
Achados oculares em crianças com toxoplasmose congênita
}

\section{Ocular findings in children with congenital toxoplasmosis}

\author{
Janer Aparecida Silveira Soares ${ }^{1}$, Luciano Sólia Násser ${ }^{2}$, Sílvio Fernando Guimarães Carvalho ${ }^{1}$, Antônio Prates Caldeira ${ }^{1}$
}

\section{RESUMO}

Objetivo: Conhecer as lesões oculares mais frequentes encontradas em crianças expostas à toxoplasmose congênita.

Métodos: Trata-se de um estudo retrospectivo, a partir de uma coorte histórica, de abordagem quantitativa. Foram avaliadas crianças encaminhadas de um serviço de infectologia pediátrica e inseridas apenas aquelas com diagnóstico confirmado de toxoplasmose congênita. A avaliação oftalmológica incluiu o mapeamento de retina sequencial, sob dilatação pupilar.

Resultados: Das 58 crianças presumivelmente expostas ao risco de doença durante a gestação, 20 apresentaram lesões oftalmológicas ao longo do primeiro ano de vida (34 olhos). Destas, 12 estavam assintomáticas ao nascimento. Estrabismo foi registrado em 14 crianças (70\%). Em uma criança observou-se ptose palpebral e em outra diminuição da fenda palpebral (microftalmia). Retinocoroidite foi a complicação mais frequente, presente em todas as 20 crianças. Sete crianças apresentaram alterações unilaterais (35\%) e 13 crianças apresentaram alterações bilaterais (65\%), prevalecendo a localização no polo posterior e mácula.

Conclusão: Retinocoroidite e estrabismo destacaram-se como importantes sequelas da toxoplasmose congênita.

Descritores: Toxoplasmose congênita; Coriorretinite; Criança; Infecções oculares parasitárias; Sinais e sintomas

\begin{abstract}
Purpose: This study aimed to know the most common ocular findings in children with congenital toxoplasmosis.

Methods: This is a retrospectivestudy carried out from a historical cohort, with a quantitative approach. We evaluated children referred to a pediatric infectious disease service and included only those with confirmed diagnosis of congenital toxoplasmosis. The ophthalmologic evaluation included regular fundus examination under pupil dilation.

Results: Of 58 children presumably exposed to risk of the disease during the pregnancy, 20 had ocular lesions during the first year of life (34 eyes). Of these, 12 were asymptomatic at birth. Strabismus was noted in 14 children (70\%). In one child there was ptosis, and another had decrease in the palpebral fissure (microphthalmia). Retinochoroiditis was the most common complication, present in all 20 children. Seven children (35\%) showed unilateral changes and 13 children showed bilateral changes (65\%), with emphasis on the location in the posterior pole and macula.
\end{abstract}

Conclusion: Retinochoroiditis and strabismus were outstanding as important sequelae of congenital toxoplasmosis.

Keywords: Toxoplasmosis, congenital; Retinochoroiditis; Child; Eye infections, parasitic; Signs and symptoms

\section{INTRODUÇÃo}

A toxoplasmose congênita é uma doença de múltiplas faces e na maioria das vezes apresenta-se assintomática ao nascimento ${ }^{(1)}$. Um dos aspectos mais graves da doença está nas lesões e sequelas oculares resultantes da retinocoroidite que pode se desenvolver em até $80 \%$ das crianças infectadas e não tratadas ${ }^{(2)}$. Estima-se que cerca de $50 \%$ de todos os casos de uveíte posterior em todo o mundo sejam decorrentes da infecção pelo T. gondii, o que torna esta a etiologia mais comum para a patologia ${ }^{(3)}$. O parasita pode ficar latente por anos e ocasionar lesões oculares em qualquer época da vida dos indivíduos contaminados ${ }^{(1,4,5)}$.

Os mecanismos que determinam o tropismo do parasita ainda não são claros $^{(1,3)}$. É descrito na literatura uma predileção do parasita pela mácula, atribuída por alguns autores à chegada do mesmo pelo nervo óptico ou artérias ciliares posteriores ${ }^{(6,7)}$. A fisiopatologia da doença está na dependência da ação direta do parasita no local da infecção devido a uma reação inflamatória e de questionável reação de hipersensibilidade do hospedeiro ${ }^{(1,3,6)}$, relacionando-se diretamente com a carga parasitária e o tipo de cepa. No Brasil a cepa Tipo I parece estar mais envolvida com os quadros de lesões oculares, enquanto na França e EUA a cepa Tipo II parece ser responsável pela maior parte dos acometimentos ${ }^{(3)}$. Trabalhos comparando lesões oculares por toxoplasmose demonstram que no Brasil as mesmas são mais graves e maiores que as encontradas na Europa e EUA ${ }^{(8)}$, talvez por um polimorfismo clonal. No Brasil ainda existem poucos estudos sobre a patologia ocular causada pelo T. gondii, especialmente quando se leva em conta a gravidade das manifestações nos recém-nascidos acometidos. É importante que os diversos centros de referência para a doença divulguem informações que permitam maior conhecimento das características desse grave e obscuro problema de saúde pública no país. Neste estudo objetivou-se descrever a frequência e as principais alterações oftalmológicas em crianças infectadas pela toxoplasmose congênita acompanhadas em um centro de referência para doenças infecciosas no norte de Minas Gerais.

\section{MÉTODOS}

Trata-se de um estudo retrospectivo, de coorte histórica, de exploratória de abordagem quantitativa, desenvolvido no Serviço de
Submitted for publication: March 22, 201

Accepted for publication: July 17, 2011

Study carried out at the Departamento de Saúde da Mulher e da Criança. Universidade Estadual de Montes Claros - UNIMONTES - Montes Claros (MG), Brazil.

Physician, Department of Pediatrics, Universidade Estadual de Montes Claros - UNIMONTES Montes Claros (MG), Brazil.

Physician, Department of Ophthalmology, Universidade Estadual de Montes Claros - UNIMONTES Montes Claros (MG), Brazil.
Funding: No specific financial support was available for this study

Disclosure of potential conflicts of interest: J.A.S.Soares, None; L.S.Nasser, None; S.F.G.Carvalho, None; A.P.Caldeira, None.

Correspondence address: Antônio Prates Caldeira. Av. Dr. Rui Braga, Prédio 6 - Campus Universitário Prof. Darcy Ribeiro - Montes Claros (MG) - 39401-089 - Brazil

E-mail: antonio.caldeira@unimontes.br 
Oftalmologia da Universidade de Montes Claros/Secretaria Municipal de Saúde de Montes Claros (MG). Foram analisadas 58 crianças (116 olhos), todas procedentes do Centro de Referência em Doenças Infecciosas de Montes Claros - MG (CERDI), no período de janeiro de 2002 a dezembro de 2010. O diagnóstico de toxoplasmose congênita foi confirmado em 21 crianças (36,2\%), que foram expostas ao T. gondii durante a gestação, sendo que 20 crianças (34 olhos) apresentaram sequelas oculares ao final do primeiro ano de vida.

A avaliação oftalmológica foi realizada por um retinólogo através do exame de mapeamento de retina e dilatação pupilar com colírio de tropicamida a 1\% associado ao ciclopentolato a 0,5\% com o oftalmoscópico binocular indireto (Heine EM 30, ômega 180 - Germany e lente 20D Ocular - USA).

Foram definidos como critérios de inclusão no estudo crianças com histórico de exposição ao T. gondii durante a gestação (triagem materna), com exames sorológicos positivos para a doença, com achados de retinocoroidite ativa ou não ao longo do primeiro ano de vida. Foram excluídas as crianças que não apresentavam lgG específica para toxoplasmose após 12 meses de idade.

A pesquisa foi submetida e aprovada pelo Comitê de Ética da Universidade Estadual de Montes Claros (UNIMONTES), através do parecer № 1495/09.

\section{RESULTADOS}

Das 58 crianças (116 olhos) expostas ao T. gondii (116 olhos), 21 tiveram o diagnóstico confirmado clinica e laboratorialmente, sendo que 20 (34 olhos) apresentaram sequelas oculares ao final do primeiro ano de vida. Destas 20 crianças, 55\% eram do sexo masculino, todas nasceram a termo (idade gestacional entre 37 e 42 semanas), mas cinco delas apresentaram baixo peso ao nascer $(<2.500 \mathrm{~g})$. Com relação às características maternas, $80 \%$ das mães eram procedentes do próprio município, 90\% residiam em área urbana e a idade média das mesmas era de 22,1 anos. Nove mães de crianças contaminadas não realizaram sorologias durante a gravidez e $80 \%$ de todas as mães não realizaram nenhum tratamento durante o pré-natal. Todos os 20 pacientes mantiveram a dosagem de IgG toxoplasmose específica após 12 meses de vida.

Em relação às manifestações clínicas (Tabela 1), 60\% das crianças apresentaram-se assintomáticas ao nascimento. O estrabismo esteve presente em 14 crianças (70\%). Ptose palpebral e diminuição da fenda palpebral foram observadas em $5 \%$ dos casos.

O exame de mapeamento de retina mostrou-se alterado em 34 olhos, sendo a retinocoroidite a complicação mais frequente, presente em $100 \%$ das crianças e com comprometimento bilateral em $65 \%$ dos casos, prevalecendo a localização no polo posterior e mácula (Tabela 2). Descolamento da retina foi observado em $10 \%$ dos casos e 35\% apresentaram nistagmo associado à retinocoroidite.

À medida que as crianças foram diagnosticadas com retinocoroidite, elas foram alocadas em um grupo de pacientes com acompanhamento perene, com orientações aos pais ou responsáveis, para avaliações periódicas a cada seis meses ou menos, dependendo da gravidade das lesões. Das 20 crianças acometidas 45\% apresentaram visão subnormal ao longo do acompanhamento, em média de 3,5 anos ( $D P \pm 2,4)$.

Em relação ao desenvolvimento neuropsicomotor, 40\% das crianças registraram algum comprometimento. A presença de calcificações cerebrais foi registrada em 50\% das crianças com retinocoroidite e entre estas, duas desenvolveram dilatação ventricular.

\section{DISCUSSÃO}

Estudos retrospectivos e observacionais demonstram que entre os recém-nascidos infectados, com doença subclínica, até 85\% desenvolvem retinocoroidite durante a infância e adolescência ${ }^{(1)}$. Este estudo, condizente com a literatura, permitiu averiguar o perfil das manifestações oftalmológicas mais comuns das crianças acometidas pelo T. gondii em um centro de referência no Norte de Minas Gerais.
As sequelas associadas com a toxoplasmose congênita e o grau de comprometimento da qualidade de vida dos pacientes infectados tornam a doença um importante problema de saúde para o Brasil. É sabido que a intervenção eficaz contra a infecção aguda pelo parasita reside no enfoque profilático e que a mesma evita em torno de $60 \%$ da priminfecção materna e o risco de doença aguda em seus conceptos ${ }^{(9,10)}$. Entretanto, as medidas preventivas que poderiam ser assumidas durante a gestação parecem negligenciadas pelos profissionais de saúde, pois se observou uma falha grave nas conduções do pré-natal, sendo que aproximadamente metade das gestantes não havia realizado investigação sorológica para a doença. O fato é mais grave quando se registra que o maior contingente das mesmas (80\%) residia em zona urbana e na cidade polo da região, onde o acesso à propedêutica é, pelo menos em tese, mais fácil.

Em conformidade com a literatura, a maioria das crianças não apresentava sinais ou sintomas clínicos sugestivos da doença ao nascimento ${ }^{(1,11)}$. Entre as alterações clínicas o estrabismo foi o achado mais comum, sendo observado em 14 crianças (70\%). Também foi a alteração que mais levou os pais das crianças a procurarem o serviço de saúde, quando o diagnóstico foi realizado após o nascimento. Este achado é semelhante a outros descritos na literatura que apontam frequências que se situam entre 33 e $76 \%(1,12,13)$. A retinocoroidite esteve presente em 100\% das crianças com alterações oculares e nistagmo em 35\% das crianças com diagnóstico de

Tabela 1. Principais alterações oftalmológicas e neurológicas em crianças com toxoplasmose congênita

\begin{tabular}{lcc}
\hline Variáveis & $\mathbf{n}$ & (\%) \\
\hline Nistagmo & 7 & \\
Presente & 13 & 35 \\
Ausente & & 65 \\
Retinocoroidite & 9 & \\
$\quad$ Lesão macular ambos os olhos & 5 & 45 \\
Lesão macular unilateral & 4 & 25 \\
Lesão periférica ambos os olhos & 2 & 20 \\
Lesão periférica unilateral & & 10 \\
Estrabismo & 5 & \\
Bilateral & 9 & 25 \\
Unilateral & 6 & 35 \\
Ausente & & \\
Visão subnormal & 9 & 45 \\
Presente & 11 & 55 \\
Ausente & & \\
Descolamento de retina & 2 & 90 \\
Presente & 18 & \\
Ausente & & 50 \\
Calcificações cranianas & 10 & 50 \\
Presente & Ausente \\
Atraso do DNPM* & 10 & 40 \\
Presente & & \\
Ausente & 8 & \\
\hline
\end{tabular}

(*) DNPM= desenvolvimento neuropsicomotor

Tabela 2. Lesões de retinocoroidite, segundo características anatômicas, em crianças com toxoplasmose congênita em um Centro de Refêrencia

\begin{tabular}{lc}
\hline Características anatômicas & Porcentagem \\
\hline Lesão macular bilateral & $45 \%$ \\
Lesão macular unilateral & $25 \%$ \\
Lesão periférica bilateral & $20 \%$ \\
Lesão periférica unilateral & $10 \%$ \\
\hline
\end{tabular}


toxoplasmose congênita, neste estudo. Resultados similares foram observados em estudo prévio em Minas Gerais ${ }^{(14)}$. Estes resultados equiparam-se também aos encontrados por Sáfadi et al., que observaram retinocoroidite em $95 \%$ das 43 crianças portadoras de toxoplasmose congênita em um serviço de referência na Santa Casa de São Paulo, bem como estrabismo, presente em 49\% das crianças e o nistagmo em $47 \%$ das mesmas ${ }^{(12)}$. Outros pesquisadores têm encontrado a retinocoroidite como o achado ocular mais frequente nas crianças acometidas pelo toxoplasma com achados variando entre $76-95 \%{ }^{(13-15)}$.

Assim como apontado por outros autores a maioria das crianças ( $n=18 ; 90 \%)$ apresentava lesões de retinocoroidite cicatrizadas já no primeiro exame e apenas duas delas apresentavam doença ativa e descolamento da retina ${ }^{(9)}$. Esta é uma complicação grave que já foi registrada com maior prevalência em outros estudos ${ }^{(16,17)}$.

Para crianças com toxoplasmose congênita as alterações neurológicas podem implicar em déficits cognitivos e atraso do desenvolvimento psicomotor. Todavia, tais danos podem ser resultantes também da limitação da acuidade visual, conforme trabalho realizado por Roizen, no qual testes psicológicos não revelaram o mesmo grau dos déficits operacionais ${ }^{(18)}$. No presente estudo não foram aplicados testes para avaliação de inteligência ou testes psicológicos de maturidade cognitiva, mas observou-se atraso do desenvolvimento ou distúrbios de comportamento em 8 (40\%) das crianças portadoras de retinocoroidite. Os autores consideram que esses achados podem ser resultantes do déficit visual, mas outros estudos serão necessários para corroborar essa hipótese.

\section{CONCLUSÕES}

A realidade das crianças acometidas neste estudo é similar a de outros estudos brasileiros, onde as lesões oculares se devem principalmente às retinocoroidites, com predileção pela região macular e onde se observa com maior frequência o acometimento bilateral com graus diversos de prejuízo da visão. Os resultados refletiram uma possível realidade local do acompanhamento pré-natal: demora na identificação das crianças expostas e atraso no diagnóstico e tratamento dos binômios. A gravidade das lesões também alerta para que os dados não sejam desconsiderados, pois demonstram um importante contingente de crianças com danos permanentes à saúde.

\section{REFERÊNCIAS}

1. Remington JS, McLeod R, Thulliez P, Desmonts G. Toxoplasmosis. In: Remington JS, Klein JO, Wilson CB, Baker CJ, editors. Infectious diseases of the fetus and newborn infant. $6^{\text {th }}$ ed. Philadelphia: Elsevier Saunders; 2006. p. 947-1091.

2. Koppe JG, Loewer-Sieger DH, de Roever-Bonnet H. Results of 20-year follow-up of congenital toxoplasmosis. Lancet. 1986;1 (8475):254-6.

3. Commodaro AG, Belfort RN, Rizzo LV, Muccioli C, Silveira C, Burnier Jr MN, Belfort Jr R. Ocular toxoplasmosis: an update and review of the literature. Mem Inst Oswaldo Cruz. 2009; 104(2):345-50.

4. Melamed J, Dornelles F, Eckert GU. Alterações tomográficas cerebrais em crianças com lesões oculares por toxoplasmose congênita. J Pediatr (Rio J). 2001;77(6):475-80.

5. Wallon M, Kodjikian L, Binquet C, Garweg J, Fleury J, Quantim C, Peyron F. Long-term ocular prognosis in 327 children with congenital toxoplasmosis. Pediatrics. 2004;113(6):1567-72.

6. Garweg JG, Candolfi E. Immunopathology in ocular toxoplasmosis: facts and clues. Mem Inst Oswaldo Cruz. 2009;104(2):211-20.

7. Gilbert RE, Freeman K, Lago EG, Bahia-Oliveira LM, Tan HK, Wallon M, Buffolano W, Stanford MR, Petersen E; European Multicentre Study on Congenital Toxoplasmosis (EMSCOT). Ocular sequelae of congenital toxoplasmosis in Brazil compared with Europe. PLoS Negl Trop Dis. 2008;2(8): e277.

8. Andrade GMQ. Triagem neonatal como estratégia para o diagnóstico e tratamento precoces da toxoplasmose congênita em Belo Horizonte, Minas Gerais (tese). Belo Horizonte: Faculdade de Medicina da Universidade Federal de Minas Gerais; 2008.

9. Gollub EL, Leroy V, Gilbert R, Chêne G, Wallon M; European Toxoprevention Study Group (EUROTOXO). Effectiveness of health education on Toxoplasma-related knowledge, behavior, and risk of seroconversion in pregnancy. Eur J Obstet Gynecol Reprod Biol. 2008;136(2): $137-45$.

10. Lopez A, Dietz VJ, Wilson M, Navin TR, Jones JL. Preventing congenital toxoplasmosis. MMWRRecomm Rep. 2000;49(RR-2):57-68. Review.

11. Andrade GMQ, Tonelli E, Oréfice F. Toxoplasmose e gestação. In: Couto JCF, Andrade GMQ Tonelli E. Infecções perinatais. Rio de Janeiro: Guanabara Koogan; 2006. p.445-71.

12. Sáfadi MA, Berezin EN, Farhat CK, Carvalho ES. Clinical presentation and follow up of children with congenital toxoplasmosis in Brazil. Braz J Infect Dis. 2003;7(5):325-31.

13. Mets MB, Holfels E, Boyer KM, Swisher CN, Roizen N, Stein L, et al. Eye manifestations of congenital toxoplasmosis. Am J Ophthalmol. 1997;123(1):1-16.

14. Santos LP, Alvarenga LS, Ferreira MA. Alterações oculares em crianças com toxoplasmose congênita precoce. Arq Bras Oftalmol. 1999;62(5):590-3.

15. McLeod R, Kieffer F, Sautter M, Hosten T, Pelloux H. Why prevent, diagnose and treat congenital toxoplasmosis? Mem Inst Oswaldo Cruz. 2009;104(2):320-44.

16. Bosch-Driessen LH, Karimi S, Stilma JS, Rothova A. Retinal detachment in ocular toxoplasmosis. Ophthalmology. 2000;107(1):36-40.

17. Lucena DR, Ribeiro JAS, Lucena DR, Lucena ALP, Jorge R. Roturas retinianas em retinocoroidite por toxoplasmose: série de casos. Arq Bras Oftalmol. 2009;72(6):829-31.

18. Roizen N, Kasza K, Karrison T, Mets M, Noble AG, Boyer K, et al. Impact of visual impairment on measures of cognitive function for children with congenital toxoplasmosis: implications for compensatory intervention strategies. Pediatrics. 2006;118(2): e379-90.

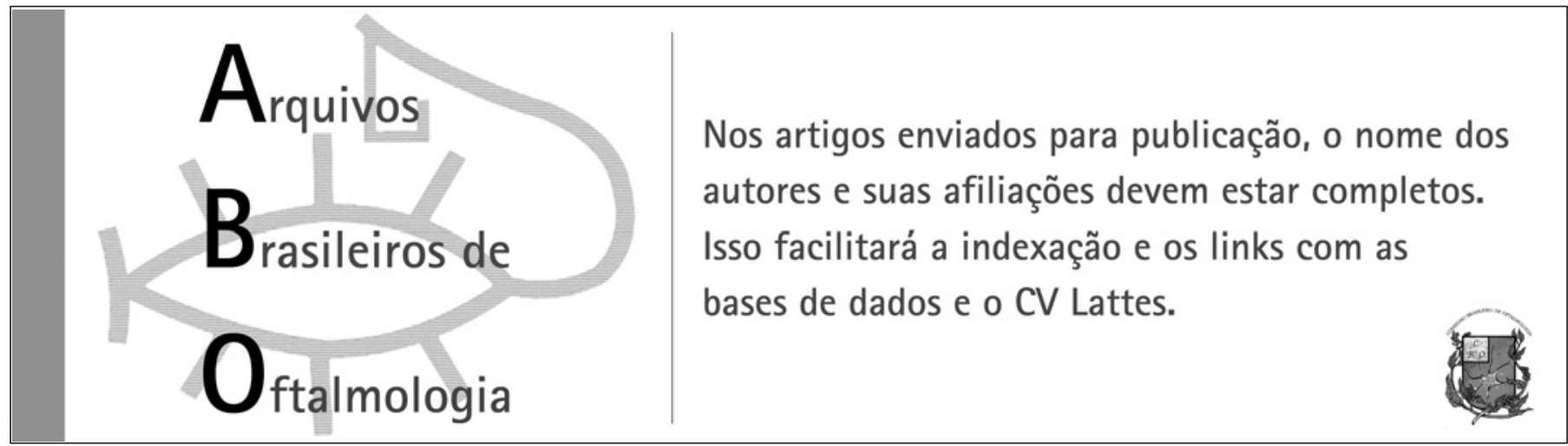

